\title{
Richard Lawrence* Giving the Value of a Variable
}

https://doi.org/10.1515/krt-2021-0007

Published online April 9, 2021

\begin{abstract}
What does it mean to 'give' the value of a variable in an algebraic context, and how does giving the value of a variable differ from merely describing it? I argue that to answer this question, we need to examine the role that giving the value of a variable plays in problem-solving practice. I argue that four different features are required for a statement to count as giving the value of a variable in the context of solving an elementary algebra problem: the variable must be in the scope opened by the problem statement; the values given must be in the range of the variable, which is determined by the problem; the statement giving the values must represent a complete solution; and it must be in a canonical form. This account helps us better understand elementary algebra itself, as well as the use of algebraic tools to analyze phenomena in natural language.
\end{abstract}

Keywords: algebra, variables and values, mathematical practice, scope, range, canonical form

\section{Algebraic Investigations}

I want to discuss an activity I call an 'algebraic investigation'. In general, an investigation is the process of answering a question. When you investigate, you start by understanding a question, and then you take certain steps to find the answer. In an algebraic investigation, the question is an elementary algebra problem, and the steps you take are the algebraic manipulations that lead you to its solution. For example, consider a simple polynomial equation of the form:

$$
x^{2}-6 x+9=0
$$

In a beginning algebra course, an equation like this is typically used to give a problem or exercise. This equation gives a problem because it describes a certain number, $x$, without specifying which number that is. The ' $x$ ' represents an epistemic gap in the statement, something unknown which must be found out. The equation therefore invokes a question: which number $x$ is such that $x^{2}-6 x+9=0$ ? In a

\footnotetext{
*Corresponding author: Richard Lawrence, Seminar für Sprachwissenschaft, Eberhard Karls Universität Tübingen, Tübingen, Germany, E-mail: richard.lawrence@uni-tuebingen.de
} 
classroom or textbook, this question might only appear in an abbreviated form, like "What is $x$ ?" or "Find $x$ ", or it might not be given explicitly at all. Nevertheless, the process of solving this problem begins from an understanding of this question. To get started in solving the problem, you need to understand that finding the answer to this question is your goal.

This goal imposes a structure on the activity of solving the problem. To reach that goal, you might proceed like this:

$$
\begin{gathered}
(x-3)(x-3)=0 \\
x-3=0 \\
x=3
\end{gathered}
$$

These manipulations-factoring, eliminating a duplicate root, and arithmetically simplifying-are the steps you take to find the answer to the question. They are appropriate in this context because they bring you closer to that goal. Many other manipulations are also possible, but unproductive: you could add 7 to both sides of these equations, for example, but that would only carry you further away from the solution. When you arrive at the last equation, you have solved the problem. This equation tells you what you didn't know at the start, namely, that 3 is the number which makes Equation (1) true. Thus, you have found the answer to the initial question, so your investigation concludes.

The main issue I want to address in this essay is: why does the investigation end exactly here? Why does writing down the Equation (4) count as solving the problem given with (1)?

It may seem that this question has an obvious answer: this equation solves the problem because it gives the value of $x$. In general, one answers questions in elementary algebra by giving the values of variables. But what does that mean? Most of us take for granted that we understand what 'giving' the value of a variable means in the context of an algebra problem; but if questioned, we'd find it difficult to say exactly what counts as doing so. Why does Equation (4) count as giving the value of $x$, for example, while (1)-(3) do not? After all, the four equations are truthconditionally equivalent, and contain exactly the same information: each is true if, and only if, the value of $x$ is 3. Yet (4) is the only line in the derivation that counts as answering the question posed with (1). What confers this special status on (4)?

My primary aim here is to provide a systematic answer to this puzzle. My account will give some criteria that an algebraic statement must satisfy for it to count as giving the value of a variable and concluding an algebraic investigation. It will depend on the idea that an algebraic investigation is a kind of inquiry, an activity structured by the goal of finding the answer to an algebraic question. As we will see, what counts as 
giving the value of a variable depends strongly on how that initial question is understood, and how subsequent steps in the investigation are seen in its light.

\section{Some Motivations}

Why is such an account interesting? What will it help us understand? First and foremost, the account I offer here will interest philosophers of mathematics, because it improves our understanding of a fundamental part of mathematical practice. Elementary algebra is the first place where students encounter the idea that variables stand for 'unknowns' whose values can only be given or specified later, as the result of further investigation. This idea is so fundamental to modern mathematics and logic that it is hard to imagine them without it. But the idea is far from an obvious one; historically, it actually took a long time to develop. ${ }^{1}$ Precisely because it is now so fundamental to our mathematical practice and education, it is worthwhile to bring its complex epistemological structure to light.

Elementary algebra is the simplest and clearest place where we find this structure, but it is hardly the only one. Any place in mathematics where we ask the question of which things satisfy a certain condition has the same structure. When we investigate questions like "What is the Galois group of this polynomial?" or "What are the bounded harmonic functions on the plane?" we go through a process similar to an algebraic investigation. ${ }^{2}$ This process begins with an understanding of the question, which asks for 'unknowns' satisfying a certain condition. The condition plays a role similar to a polynomial equation like (1): it describes certain values without specifying them. The investigation proceeds by searching for these values, and ends by giving a specification of exactly which values they are. The search will use different methods than those of elementary algebra, and the answer will be expressed in different ways, but the basic structure is the same.

Of course, there are other kinds of questions in mathematics, such as yes-no questions like "Are there functions which are nowhere differentiable but everywhere continuous?” or “Are there any primes between $2^{256}$ and $2^{257}$ ?” We can often investigate whether something is true without asking which things make it true, and in such cases, the investigation has a different epistemological structure. ${ }^{3}$

1 For a fascinating history of how this central algebraic idea developed and how it enabled so much of modern mathematics, (see Derbyshire 2006).

2 Thanks to an anonymous referee and Peter McGrath for these examples.

3 Belnap and Steel (1976) recognize (wisely, I think) a fundamental distinction between whichquestions and whether-questions, because they have different sorts of possible answers, and so different processes are required to answer them. The questions which give rise to investigations with the structure I describe here are all which-questions, in their sense. 
Still, for a large class of mathematical questions, elementary algebra serves as a particularly clear model of how investigations into these questions proceed. My account of what it means to give the value of a variable in elementary algebra readily extends to these other cases, so it can help us understand a widespread phenomenon in the epistemology of mathematics.

Indeed, the account is also of more general interest, because this same epistemological structure appears outside of mathematics. Investigations with this structure are pervasive in both scientific practice and in ordinary life, and algebraic variables play an important role in our understanding of the language used to carry them out. Suppose, for example, that I say:

A man walked in.

Or suppose, observing the same scene, you ask:

Who walked in?

The indefinite description "a man" in my statement, as well as the question word "who" in your question, will typically be analyzed as introducing a variable. ${ }^{4}$ That's because both sentences describe a certain person who remains 'unknown': in much the same way that Equation (1) describes a number without specifying which number it is, these sentences tell us that a person walked in without specifying which person it was. ${ }^{5}$ A variable is an appropriate choice for representing the semantic contribution of these expressions because both sentences invite further investigation to find out who that unknown person is, just like a polynomial equation.

Natural language also contains sentences that we might think of as giving the values of such variables. If the next statement in the context is

It was George.

Or, more explicitly,

The man who walked in was George.

then what was unknown becomes known. These sentences feel very much like Equation (4): they specify the person in question, so they seem to give the value of

4 The analysis of indefinites as introducing variables bound by a quantifier traces back to Russell's analysis of descriptions (Russell 1905), and has become completely standard; see also the analysis in (Heim 2002), which treats indefinites as unbound variables. The use of variables to analyze question words also has a long tradition and has become standard (Belnap and Steel 1976; Groenendijk and Stokhof 1997; Hamblin 1973).

5 The question tells us this by presupposing that someone walked in, rather than by asserting it. 
the variable introduced earlier. ${ }^{6}$ They conclude the investigations that the earlier sentences invite. But without a systematic account of what it means to give the value of a variable, we can't yet fully exploit the analogy with algebra to understand their internal semantics, or their relationship to expressions like "a man" or "who" in prior discourse. Relatedly, we might say that someone who hears the above exchange "knows who walked in". An account of what it means to give the value of a variable is needed to analyze this ascription of knowledge, and to explain the relationship between knowing who walked in and knowing that George walked in. ${ }^{7}$

Of course, the same phenomena arise in scientific uses of natural language. Consider a chemist who says: "A strange compound precipitated out during the experiment this morning” and later reports: "It was arsenic sulfide.” Or consider an astronomer who wonders which pair of galaxies she is looking at, and later determines that it was NGC 2207 and IC 2163. In all such cases, an investigator starts from a question about which unknown thing or things meet a certain condition, and through investigation comes to know and to say which things those are; and this process can be looked at as finding out and giving the value of a variable. A secondary aim of this essay, then, is to provide the foundations which support such applications of algebraic variables to the analysis of natural and scientific language, and to the shifts in our knowledge that such language is used to express. Though I will not say more about these applications here, I have looked at them in more detail elsewhere. ${ }^{8}$

6 Such sentences are known in linguistics as specificational sentences. When linguists characterize them, they often spontaneously resort to a comparison with equations like (4). Mikkelsen (2011, pp. 1809-1810) writes, for example, that in specificational sentences, the left side "introduces a variable", while the right side "provides the value for that variable". Similar remarks appear in much of the linguistics literature on specificational sentences (e.g., Akmajian 1970; Heycock and Kroch 1999; Higgins 1979). Recently, philosophers have become interested in specificational sentences in the context of a debate about how to analyze sentences like "The number of Jupiter's moons is four” (Brogaard 2007; Felka 2014, 2016; Knowles 2015; Moltmann 2013; Snyder 2017).

7 This relationship has been important in recent discussions about knowledge-wh (Parent 2014). The main issue in those discussions is how to understand claims of the form " $S$ knows $Q$ ", where $Q$ is an interrogative formed with a wh-word. Reductionists hold that such claims are true just in case $S$ knows that $p$, where $p$ is the (or a) true answer to $Q$ (see e.g. Boër and Lycan 1986; Hintikka 1975; Stanley and Williamson 2001). A number of authors have recently challenged reductionism, arguing that knowledge-wh ineliminably involves the question $Q$ (Brogaard 2009; Farkas 2016; Masto 2010; Schaffer 2007). In one typical kind of case, $p$ answers $Q$ by virtue of having definite values in the places where $Q$ has variables, so understanding what it means to give the value of a variable is an important preliminary step toward resolving the debate over reductionism about knowledge-wh.

8 See Lawrence (2017). 


\section{Four Criteria for Concluding an Algebraic Investigation}

Let's now turn to the details of the account. We have seen that when an equation like (4) gives the value of a variable, it has a special status: it marks the end of an algebraic investigation, the point at which the goal is reached and the question is answered. What confers this special status on (4), in contrast to Equations (1)-(3) in the derivation above? That is, why does writing down this equation, in this context, have the epistemological significance of concluding the investigation-as the other lines do not, and as the same equation would not in a different context? This is the question to be addressed here.

As I have already noted, each line of this derivation is truth-conditionally equivalent to the others. Thus, to understand why only the final equation counts as giving the value of $x$, we need to look beyond its truth conditions. For its truth conditions are the same as the other equations', but we need to know what makes it different: we need to explain why deriving this final equation counts as discovering the value of $x$, and therefore as coming to know the answer to the algebraic question, but deriving the earlier equations does not. What else, then, could explain this equation's unique epistemic role?

Here is my answer, in outline. There are four different features of Equation (4) which are crucial for it to count as giving the value of $x$ and as answering the question invoked by (1). These are: that the variable is in the same scope as the variable introduced by the problem statement; that the value given is in the range of the variable; that the equation represents a complete answer to the question; and that the equation is in a canonical form. These are not features of the equation itself; they are features of how it is used and understood in this particular context. Each of them is individually necessary for this equation to be understood as giving the value of $x$. Together, they provide a reasonably complete account of why this equation answers the problem, but the earlier equations do not. Let me describe each in turn.

\subsection{Scope}

It is easiest to see what I mean by 'scope' if we ask: why does the equation $x=3$, as it is used on line (4), give the solution to the problem given on line (1), as opposed to some other problem? The obvious answer is that the equations on these two lines of the derivation employ the same variable. But what is it for the variable to be 'the same' in these two lines? This is obviously not a purely typographical matter. The 
symbol ' $x$ ' can be used to express a variable in many different problems. I might introduce a new problem to you with an equation like

$$
x^{2}-4 x+4=0
$$

without causing any confusion. Students of elementary algebra have to learn that the symbol ' $x$ ' is being used differently here than in (1), and that its use in (4) is connected with its use in (1) but not in (5). This is what I mean by scope: a variable's scope includes just those statements in which its occurrences are understood to be connected.

In elementary algebra, a variable's scope thus depends on the problemsolver's understanding of her own activity. Its scope extends exactly as far as the statements belonging to a single algebraic investigation, and no further. This is because an algebraic variable originates as an 'unknown' with the question that structures the investigation. Understanding a statement containing a variable requires recognizing it as part of an attempt to answer that question by finding the variable's value. A variable's scope thus opens with a posing of a problem, using one or more equations; it closes with a statement of its solution, which gives the value (or values) of that variable; and it includes whatever statements occur between the problem statement and the solution statement that are part of the problem-solver's effort to move from the former to the latter.

There is no sense in asking for the value of a variable apart from some scope or other. A variable only has a value relative to the question which introduces it. To say that an equation gives the value of a variable is therefore to connect that equation with an attempt to answer a particular problem or question. ${ }^{9}$ It is to place the use of that equation in the context of some algebraic investigation or other. This gives us our first criterion: for an equation to count as giving the value of a variable,

9 Thus, in the language used by authors like Schaffer (2007), Masto (2010) and Farkas (2016), I am proposing an 'anti-reductionist' view of the knowledge acquired in an algebraic investigation. Anti-reductionism is the view that the question plays an essential role in characterizing this knowledge: the student who derives (4) does not simply come to know that $x=3$, but comes to know it as the answer to the question of which number $x$ is such that $x^{2}-6 x+9=0$. Apart from the context of some such question, it makes no sense to say that someone comes to know 'that $x=3$ '. A key argument for anti-reductionism is the observation that, since the same proposition $p$ can answer different, non-equivalent questions, knowing that $p$ must be compatible with having knowledge-wh of one these questions without the others. In the present context, this corresponds to the observation that deriving an equation that gives the value of a variable only counts as coming to know the answer to the algebraic question in whose scope it occurs. The student who derives (4) from (1) knows which number $x$ is such that $x^{2}-6 x+9=0$, but not which number $x$ is such that $x^{3}-9 x^{2}+27 x=27$, even though $x=3$ in both cases. 
the variable in that equation must occur in the same scope as in the problem to which the equation gives the solution.

\subsection{Range}

Of course, the problem which introduces a variable must actually have a solution if its value is to be given. This depends on a feature of how the problem is understood: the variable's range. Take, for example, the problem given by the following equation:

$$
x^{2}+5 x+6=0
$$

If $x$ is taken to range over the natural numbers in this problem, then it has no solution, as you can see once it has been factored:

$$
(x+2)(x+3)=0
$$

The roots of (6) are -2 and -3 . They are both negative integers, so they are not among the natural numbers. So whether or not this problem has solutions depends on whether $x$ ranges over just the natural numbers, or over some more inclusive set, like the integers. It is only possible to give a value for $x$ in this case when $x$ is understood as ranging over some set that includes at least -2 or -3 . Different understandings of the range of a variable thus influence whether, and how, a value for that variable can be given. This gives us a second criterion: for an equation to count as giving the value of a variable, that variable must be understood to have a certain range, and the value given must fall within that range.

\subsection{Completeness}

The previous example raises another important point. Many polynomial equations, unlike (1), have more than one root, at least on some ways of understanding the range of the variable. For this reason, it is in general too simple to ask under what conditions a statement gives the value of a variable. When $x$ is understood to range over the integers in (6), an equation like

$$
x=-2
$$

could be said to give 'a' value of $x$, but not 'the' value of $x$, since no value in that range uniquely satisfies the equation. For an algebraic statement to count as a solution statement, it must give at least one value for the problem's variable. But in 
general, there is a further question as to how many such values must be given for the end of an algebraic investigation to be attained.

For equations with multiple distinct roots, we need to distinguish among several possible understandings of the problem in order to answer this question. Because it has multiple roots, an equation like (6) actually underdetermines which question is being asked. If the question is "What are all the numbers $x$ such that $x^{2}+5 x+6=0$ ?", then a complete answer must give both roots. Someone solving this problem would have to write something like

$$
x=-2 \text { or } x=-3
$$

to completely answer this question and achieve the end of her investigation. On the other hand, if the question is "What is one number $x$ such that $x^{2}+5 x+6=0$ ?" then

$$
x=-3
$$

gives a complete answer to the problem. Even though it does not exhaustively list the roots of the equation, the problem-solver can stop at this point, since this equation provides everything the question asks for.

Borrowing some terminology from Belnap and Steel (1976), we can say that although these two problems are posed with the same polynomial, they make different requests. A problem's request determines how many solutions an answer should provide, and whether a correct answer should provide an exhaustive list of them. In general, the answer to the question that initiates an algebraic investigation is not complete unless it fulfills this request, so what counts as completely solving a problem depends on how the problem's request is understood.

This shows that we should distinguish giving a value of a variable from giving the answer to an algebraic question. Depending on the problem's request, it may be necessary to give more than one value for a variable in order to give a complete answer. The same distinction is also needed when we look the more general class of algebra problems given by systems of equations in multiple variables, such as the problem given by (10) and (11):

$$
\begin{gathered}
x^{2}+y^{2}=25 \\
x-y=1
\end{gathered}
$$

Here is one solution to this problem:

$$
x=4, y=3
$$

This solution statement contains two equations as parts, each of which gives a different value for a different variable. Giving a value for a variable is necessary, 
but not sufficient, for completing an algebraic investigation. A problem-solver only completes an investigation when she gives an answer that provides as many solutions as requested, each of which must give values for each of the variables in the problem.

\subsection{Canonical Form}

The final criterion concerns which kinds of expressions are suitable for giving the value of a variable. I call these criteria of canonical form. When an equation is suitable for giving the value of a variable, it must also be in canonical form. To see the importance of canonical form, consider again the final two lines from the derivation in (1)-(4):

$$
\begin{gathered}
x-3=0 \\
x=3
\end{gathered}
$$

As we noted earlier, these equations are equivalent. What then is the difference between them? Why is it that only the second of these equations counts as giving the value of $x$, and thereby giving the solution to the problem in (1)?

The point I want to emphasize here is that some standard or other is always required in a problem-solving practice to distinguish among equations like these. Part of understanding a problem is grasping the criteria that distinguish those statements which can be used to give values for the problem's variables from those which, though otherwise equivalent, cannot. In the absence of such criteria, we have no grounds for distinguishing equations that are used to give problems from those that are used to give solutions, and so no possibility of a problem-solving activity at all. Thus, for an equation to count as giving a value of a variable, some such criteria must be in place, and the equation must fulfill them.

What are those criteria? Looking at the two equations above, a natural first thought is that the second equation is syntactically and arithmetically simpler than the first. This can't be the whole story about canonical form, though, because there are other pairs of equations in which one equation is no simpler than the other, but only one counts as giving the value of $x$. Here are two examples:

$$
\begin{array}{ll}
2 x=1 & -x=2 \\
x=\frac{1}{2} & x=-2
\end{array}
$$

At least in the classrooms where I was taught, only the lower equation in each pair would have counted as a complete solution to a problem, even though it is no syntactically or arithmetically simpler than the upper one. 
Usually, we can say that for an equation to be in canonical form, the variable should be arithmetically isolated, and purely numerical expressions should be fully computed. But even these generalizations are not exceptionless, and apart from them, whether an expression counts as being in canonical form is a fairly local matter. Consider, for example, the problem given by

$$
18 x=8
$$

Here are two possible ways of writing a solution to this problem:

$$
x=\frac{4}{9} \quad x=0 . \overline{4}
$$

Neither of these equations is 'more canonical' than the other in any global sense. A teacher who assigns this problem as an exercise might accept one equation as a solution but not the other depending on his pedagogical purposes. For example, he might accept the right-hand equation as a complete solution, but the left-hand equation as only a partial solution, in a lesson emphasizing decimal expansions of rational numbers. In a lesson emphasizing exact expressions of ratios, or where calculators are not permitted, the standard might be the other way around.

Criteria of canonical form, then, are inherently local and purpose-relative, even if there are some general rules that apply in most problem-solving settings. So what, if anything, unifies the various standards we might have? Is there any general account of what it is for an expression to be in canonical form?

Here is my answer: a standard of canonical form determines a distinguished class of expressions such that, when one of these expressions is used to give the value of a variable, no further question can be appropriate about which value is meant. This becomes clearer if we imagine an algebraic investigation formulated as an explicit question and a series of attempted answers:

Which number $x$ is such that $4 x^{2}-4 x+1=0$ ?

If you were to ask someone this question, it would obviously be unsatisfying to be told, in reply, that

It is the number $x$ such that $4 x^{2}-4 x+1=0$.

even though this answer is in a sense perfectly true. This 'answer' merely repeats the problem; it is unsatisfying because it invites the rejoinder, "But which number is that? That's what I wanted to know.” It would only be slightly more satisfying if the reply was

It is the number $x$ such that $(2 x-1)^{2}=0$. 
Here again, it can be appropriate to ask "But which number is that?", and insofar as it is, the respondent has not fully answered the original question. ${ }^{10}$ But this question is no longer appropriate if the respondent says:

It is the number $\mathrm{x}$ such that $x=\frac{1}{2}$.

A further 'Which number is that?' question in this case would indicate a misunderstanding on the part of the questioner. Our practices of giving numbers simply do not allow any further request here; the expression ' $x=\frac{1}{2}$ ' makes it as clear which number is meant as anyone has any right to demand. This equation, unlike those in the other answers, is in canonical form. Its being in canonical form just consists in the fact that no such demand is appropriate.

I am not the first to emphasize the importance of canonical form. In an unpublished lecture, Saul Kripke describes a closely related concept, which he calls a buck-stopper. For Kripke, a buck-stopper is a term such that "the question 'Yes, but which [number is that] and how did I know this?' is completely idle, and no answer to it need be given" (Kripke 1992, p. 15). Thus, when an equation is in canonical form in my sense, the term within it that specifies the variable's value is a buck-stopper for Kripke, like ' $\frac{1}{2}$ ' in the example just given. Similar concepts appear in discussions by other authors, too. Kripke cites Ackerman (1978), who calls numerals standard names for numbers, and contrasts their behavior under propositional attitude verbs with complex arithmetical terms and descriptions like ' $8+3$ ' or 'the least perfect number'. Dummett likewise suggests that a "preferred range of names" is needed to ground the (Fregean) senses of terms that refer to numbers and other abstract objects (Dummett 1981, p. 499). More recently, Heylen (2014) speaks of the Peano numerals as canonical terms for numbers, and argues that their special status is explained by the induction schema in Peano arithmetic. These authors all suggest, in different ways, that a certain subclass of terms for numbers play a special role in our epistemic relations to them and our language for reporting those relations.

I agree with these authors that such canonical ways of giving numbers are crucial for mathematics. What is missing in their discussions, I think, is an

10 Authors like Boër and Lycan (1986) and Aloni (2005) share my intuition here that an unsatisfying reply to such a question does not count as an answer even if it is true, and thus that answerhood depends on contextual features like the purposes of the questioner. A contrary view is offered by Braun (2006), who distinguishes the notion of answering a question from that of satisfying the inquirer. On Braun's view, we should say that these replies answer the question but are unsatisfying. But as Masto (2010) notes, Braun's invariantist understanding of the questionanswer relation won't help us understand an inquiry like an algebraic investigation as a process of making progress toward finding an answer, since even a trivial, unsatisfying answer counts as an answer on Braun's view. 
awareness of the crucial role of canonical terms in investigations. These discussions have tended to focus on the natural numbers and our numerals for them, and to explain the special status of these numerals by reference to the fact that they form a recursively enumerable set. But the felt contrast between canonical and non-canonical terms is not limited to this (fairly unusual) case. We can still draw the distinction between canonical and non-canonical terms in algebraic investigations where the range of a variable is not enumerable: we have both canonical and non-canonical terms for transcendental numbers like $\pi$ and $e$, for example. We also feel a similar contrast in other problem domains. Consider a question like: "What is the group of symmetries of a regular tetrahedron?" and the contrast between answers like ' $S_{4}$ ' and 'the symmetric group with 30 subgroups'. Or consider again a question in ordinary language like "Who walked in?" and the contrast between replies like 'George' and 'the only person I saw yesterday'. For any question that asks for a specification of the values satisfying a certain condition, we can draw a contrast between answers which permit a rejoinder of "But which one is that?" and answers which don't, even if we have no way of enumerating all and only the latter answers.

The special status of these terms has less to do with their enumerability and more to do with the epistemological structure of investigations in general. From the perspective I have offered here, the crucial feature of standards of canonical form is that they allow us to recognize when a process of inquiry has come to an end. When we start an investigation into a particular question, we don't know what the answer is (if we did, there would be no point in investigating!). But unless we have at least some idea of what the answer must be like, the investigation could go on forever: without some way of distinguishing an answer from what is merely a step toward an answer, we will have no way to recognize an answer when we finally find it. It is true that recursively enumerable sets of terms, like numerals for the natural numbers, provide us with an especially clear idea of what the answers to many algebraic investigations should be like. But as I have indicated, our standards of canonical form may still vary from one problem-solving context to the next. The important point is that some such standards are always in play during an algebraic investigation, because it is by understanding and applying them that we recognize that the problem has been solved, and the investigation is concluded.

\section{Conclusions}

Here is a summary of the above observations. Algebraic practice is a practice of investigation, of posing questions and seeking their answers. When we say that an equation like 


$$
x=3
$$

'gives the value of a variable', we are assigning it a certain role within that practice. We are saying it is part of a solution statement, which concludes an algebraic investigation by fulfilling its aim. It forms part of the answer to the "Which numbers $x$ are such that ...?” question that structures the investigation, by specifying one of the values that this question asks for. This distinguishes it from equations which state problems, or mark intermediate steps between a problem and a solution, which do not give a value for the variable and cannot be part of a solution statement.

In order for an equation to play that role, it must be understood in a certain way. Its variable must be in the same scope as the variable introduced by the problem statement, and it must give a value within the range that the variable is understood to have. To count as giving a value, rather than just describing it, the equation must satisfy some criteria for being in canonical form, using an expression for the value which is not subject to a "But which do you mean?" rejoinder by the standards of the problem-solving practice. When a polynomial used to pose a problem has more than one root, or contains more than one variable, more than one such equation might be necessary to solve the problem and complete the investigation. How many such equations are necessary is a matter of how the question structuring the investigation is understood: how many variables it introduces, how many solutions it requests, and whether or not it requests an exhaustive list of solutions.

Through an algebraic investigation, one comes to know which number (or numbers) make some polynomial equation (or system of equations) true. On the view I have offered, this knowledge is best characterized in relation to the question which opens the investigation; what one comes to know depends on how exactly that question is understood. In this sense, knowing which number $x$ is such that $x^{2}-6 x+9=0$ is different from simply knowing that $3^{2}-6 \cdot 3+9=0$. One can come to know the latter without going through an algebraic investigation-say, by playing idly with a calculator. But in that case, there is no need to characterize the knowledge in terms of the question it resolves, and precisely for that reason, there is no need to describe the acquisition of such knowledge as a process of finding out the value of a variable. In elementary algebra, a variable is a device which links the giving of a value back to a question that asks which values it has. When we describe someone as knowing which number $x$ is such that $x^{2}-6 x+9=0$, or as knowing that $x=3$, we characterize her as knowing something as the answer to a prior algebraic question. Her knowledge is the outcome of an investigation.

As I indicated at several points, the account I have given is also useful for understanding other kinds of investigations, including other problems in 
mathematics, as well as investigations in science and everyday life. It makes sense to look at many different investigations as a process with a structure similar to an elementary algebra problem: it begins with a question that introduces one or more variables as 'unknowns', and ends by giving the values of these variables, that is, by specifying what was previously unknown. If we extend the account developed here to this wider class of cases, many interesting further questions arise. For example: what does it mean to understand the range of the variable in a question like "Under what conditions can a planet support life?" How do we recognize the scope of an investigation into this question, where the statements which belong to it might be made over long distances in space or time, and by many people? How do we converge on standards of canonical form that help us recognize when the investigation is completed? The account offered here does not answer these questions; but part of its interest is that it helps bring them to light.

\section{References}

Ackerman, D. 1978. “De Re Propositional Attitudes Toward Integers." The Southwestern Journal of Philosophy 9 (2): 145-53.

Akmajian, A. 1970. Aspects of the Grammar of Focus in English. Cambridge: Massachusetts Institute of Technology.

Aloni, M. 2005. “A Formal Treatment of the Pragmatics of Questions and Attitudes." Linguistics and Philosophy 28 (5): 505-39.

Belnap Jr., N. D., and T. B. Steel Jr. 1976. The Logic of Questions and Answers. New Haven, Connecticut: Yale University Press.

Boër, S., and W. Lycan. 1986. Knowing Who. Cambridge, Massachusetts: MIT Press.

Braun, D. 2006. "Now You Know Who Hong Oak Yun Is." Philosophical Issues 16: 24-42.

Brogaard, B. 2007. "NumberWords and Ontological Commitment." The Philosophical Quarterly 57 (226): 1-20.

Brogaard, B. 2009. "What Mary Did Yesterday: Reflections on Knowledge- Wh.” Philosophy and Phenomenological Research 78 (2): 439-67.

Derbyshire, J. 2006. Unknown Quantity: A Real and Imagined History of Algebra. London: Atlantic Books.

Dummett, M. 1981. Frege: Philosophy of Language, 2nd ed. Cambridge, Massachusetts: Harvard University Press.

Farkas, K. 2016. “Know-Wh Does Not Reduce to Know-That.” American Philosophical Quarterly 53 (2): 109-22.

Felka, K. 2014. “Number Words and Reference to Numbers." Philosophical Studies 168 (1): 261-82.

Felka, K. 2016. Talking about Numbers: Easy Arguments for Mathematical Realism, Vol. 3. Studies in Theoretical Philosophy. Frankfurt: Vittorio Klostermann.

Groenendijk, J., and M. Stokhof. 1997. “Questions.” In Handbook of Logic and Language, edited by J. van Benthem, and A. ter Meulen, 1055-124. Cambridge, Massachusetts: MIT Press.

Hamblin, C. L. 1973. “Questions in Montague English.” Foundations of Language 10 (1): 41-53. 
Heim, I. 2002. "File Change Semantics and the Familiarity Theory of Definiteness." In Formal

Semantics: The Essential Readings, edited by P. Portner, and B. Partee, 223-48. Oxford:

Blackwell.

Heycock, C., and A. Kroch. 1999. "Pseudocleft Connectedness: Implications for the LF Interface Level.” Linguistic Inquiry 30 (3): 365-97.

Heylen, J. 2014. "The Epistemic Significance of Numerals." Synthese 198: 1019-45.

Higgins, F. R. 1979. The Pseudo-cleft Construction in English. Outstanding Dissertations in Linguistics. New York: Garland Publishing.

Hintikka, J. 1975. "Different Constructions in Terms of the Basic Epistemological Verbs: A Survey of Some Problems and Proposals." In The Intensions of Intentionality and Other New Models for Modalities, 1-25. Dordrecht: D. Reidel.

Knowles, R. 2015. “What 'the Number of Planets Is Eight' Means.” Philosophical Studies 172 (10): 2757-75.

Kripke, S. 1992. "Logicism, Wittgenstein, and de Re Beliefs about Numbers." In Alfred North Whitehead Lectures. Cambridge, Massachusetts: Harvard University.

Lawrence, R. 2017. Nominalization, Specification, and Investigation. Berkeley, California: University of California, Berkeley.

Masto, M. 2010. "Questions, Answers, and Knowledge-Wh.” Philosophical Studies 147 (3): 395-413.

Mikkelsen, L. 2011. “Copular Clauses.” In Semantics: An International Handbook of Natural Language Meaning, Vol. 2, edited by K. von Heusinger, C. Maienborn, and P. Portner, 1805-29. Berlin: Mouton de Gruyter.

Moltmann, F. 2013. “Reference to Numbers in Natural Language.” Philosophical Studies 162: 499-536.

Parent, T. 2014. “Knowing-Wh and Embedded Questions." Philosophy Compass 9 (2): 81-95.

Russell, B. 1905. “On Denoting.” Mind 14 (56): 479-93.

Schaffer, J. 2007. "Knowing the Answer." Philosophy and Phenomenological Research 75 (2): 383-403.

Snyder, E. 2017. “Numbers and Cardinalities: What's Really Wrong with the Easy Argument for Numbers?" Linguistics and Philosophy 40 (4): 373-400.

Stanley, J., and T. Williamson. 2001. “Knowing How.” The Journal of Philosophy 98 (8): 411-44. 